\title{
High-scale leptogenesis with three-loop neutrino mass generation and dark matter
}

\author{
Pei-Hong Gu \\ Department of Physics and Astronomy, Shanghai Jiao Tong University, \\ 800 Dongchuan Road, Shanghai 200240, China \\ E-mail: peihong.gu@sjtu.edu.cn
}

ABSTRACT: We demonstrate a common origin for high-scale leptogenesis and three-loop neutrino mass generation. Specifically we extend the standard model by two real singlet scalars, two singly charged scalars carrying different quantum numbers under certain global symmetry and two or more singlet fermions with Majorana masses. This global symmetry is only allowed to be softly or spontaneously broken. Our model also respects an exactly conserved $Z_{2}$ discrete symmetry. Through the real scalar decays and then the charged scalar decays, we can obtain a lepton asymmetry stored in the standard model leptons. This lepton asymmetry can be partially converted to a baryon asymmetry by the sphaleron processes. The interactions for this leptogenesis can also result in a three-loop diagram to generate the neutrino masses. The lightest singlet fermion can keep stable to serve as a dark matter particle.

Keywords: Beyond Standard Model, Cosmology of Theories beyond the SM, Neutrino Physics

ArXiv EPrint: 1611.03256 


\section{Contents}

1 Introduction 1

2 The model 2

3 Neutrino masses and dark matter 3

4 Leptogenesis 4

$\begin{array}{lll}5 & \text { Summary } & 7\end{array}$

\section{Introduction}

The phenomena of neutrino oscillations have been established by the atmospheric, solar, accelerator and reactor neutrino experiments. This means the existence of three flavors of massive and mixing neutrinos which are beyond the standard model (SM) [1]. Meanwhile, the cosmological observations stringently constrain the neutrino masses should be in the sub-eV region [1]. Currently the most popular scheme for the neutrino mass generation is the famous seesaw [2-13] mechanism which can highly suppress the neutrino masses by a small ratio of the electroweak scale over a newly high scale. The seesaw scale can be lowed if we do some fine tuning on the related couplings. In the usual seesaw models, the leptonnumber-violating interactions for the neutrino mass generation can also accommodate a leptogenesis [14-27] mechanism to generate the baryon asymmetry in the universe. In this scenario the seesaw and the leptogenesis are realized at a same scale.

Alternatively, some $\mathrm{TeV}$-scale fields can help us to obtain the small neutrino masses at loop level [28-39]. In this scenario, the neutrino masses may be suppressed by the charity besides the loop factors. For example, Krauss, Nasri and Trodden (KNT) ever proposed an interesting model with two TeV-scale singly charged scalars and one Majorana singlet fermion to give the neutrino masses at three-loop level [32]. The Majorana singlet fermion can keep stable to serve as a dark matter particle. In order to fulfill the neutrino oscillation data which require at least two nonzero neutrino mass eigenvalues, the KNT model should contain two or more Majorana singlet fermions [33]. Although the KNT model has an advantage of testability at colliers, it cannot explain the cosmic baryon asymmetry.

In this work we will slightly extend the KNT model by two real singlet scalars in order to demonstrate an interesting scenario that a high-scale leptogenesis can be consistent with a testable neutrino mass generation. Specifically, the real singlet scalars are very heavy so that their decays can be responsible for the leptogenesis. Meanwhile, we can obtain the KNT model by integrating out these real singlet scalars. 


\section{The model}

We denote the non-SM fields by

$$
N_{R}(1,1,0)(0), \quad \delta(1,1,+1)(-2), \quad \xi(1,1,+1)(-1), \quad \sigma(1,1,0)(0) .
$$

Here and thereafter the first brackets following the fields describe the transformations under the $\mathrm{SU}(3)_{c} \times \mathrm{SU}(2)_{L} \times \mathrm{U}(1)_{Y}$ gauge groups, while the second brackets are the lepton numbers. We assume the lepton number can be softly broken. Furthermore, our model respects a $Z_{2}$ discrete symmetry under which the fields transform as

$$
(\mathrm{SM}, \delta) \stackrel{Z_{2}}{\longrightarrow}(\mathrm{SM}, \delta),\left(N_{R}, \xi, \sigma\right) \stackrel{Z_{2}}{\longrightarrow}-\left(N_{R}, \xi, \sigma\right)
$$

The $Z_{2}$ symmetry will not be broken at any scales. This means the real singlet scalars $\sigma$ will not be allowed to obtain any non-zero vacuum expectation values.

Under the softly broken lepton number and the exactly conserved $Z_{2}$ discrete symmetry, the Lagrangian should include

$$
\begin{aligned}
\mathcal{L} \supset & -\frac{1}{2} M_{\sigma_{i}}^{2} \sigma_{i}^{2}-\left(\mu_{\delta}^{2}+\lambda_{\delta \phi} \phi^{\dagger} \phi\right) \delta^{\dagger} \delta \\
& -\left(\mu_{\xi}^{2}+\lambda_{\xi \phi} \phi^{\dagger} \phi\right) \xi^{\dagger} \xi-\frac{1}{2} M_{N_{i}}\left(\bar{N}_{R i} N_{R i}^{c}+\text { H.c. }\right) \\
& -\left[\rho_{i} \sigma_{i} \xi^{\dagger} \delta+\frac{1}{2}\left(f_{\delta}\right)_{\alpha \beta} \delta \bar{l}_{L \alpha}^{c} i \tau_{2} l_{L \beta}+\left(f_{\xi}\right)_{\alpha i} \xi \bar{e}_{R \alpha}^{c} N_{R i}+\text { H.c. }\right] \\
& -y_{\alpha}\left(\bar{l}_{L \alpha} \phi e_{R \alpha}+\text { H.c. }\right) .
\end{aligned}
$$

Here $\phi$ denotes the SM Higgs scalar while $l_{L}$ and $e_{R}$ are the SM leptons,

$$
\begin{aligned}
\phi\left(1,2,+\frac{1}{2}\right)(0) & =\left[\begin{array}{c}
\phi^{+} \\
\phi^{0}
\end{array}\right], \\
l_{L \alpha}\left(1,2,-\frac{1}{2}\right)(+1) & =\left[\begin{array}{c}
\nu_{L \alpha} \\
e_{L \alpha}
\end{array}\right], \\
e_{R \alpha}(1,1,-1)(+1) \quad(\alpha & =e, \mu, \tau) .
\end{aligned}
$$

Obviously, the singlet fermions $N_{R i}$ can form the Majorana fermions as follows,

$$
N_{i}=N_{R i}+N_{R i}^{c}=N_{i}^{c}
$$

In addition, the two cubic parameters $\rho_{1,2}$ are always allowed to have a relative phase. As we will show later this phase provides the necessary $\mathrm{CP}$ violation for the leptogenesis.

So far we have assumed that the cubic terms among the non-SM scalars, i.e. the $\rho_{i^{-}}$ terms in eq. (2.3), are the unique source for the lepton number violation. Alternatively, we can consider the lepton number assignment in the KNT model where the Majorana mass term of the singlet fermions $N_{R}$ is the unique source of the lepton number violation. In this case, the present lepton number assignment in eq. (2.1) should be understood as 
an additionally global symmetry under which the SM leptons carry a quantum number as same as their lepton number. Clearly, such soft lepton number breaking or other soft symmetry breaking can be induced by a spontaneous symmetry breaking. In this case, we need introduce a complex singlet scalar,

$$
\omega(1,1,0)(+1)
$$

and then replace the $\rho_{i}$-terms in eq. (2.3) by the following quartic terms,

$$
\mathcal{L} \supset-\lambda_{i} \sigma_{i} \xi^{\dagger} \delta \omega+\text { H.c. . }
$$

After the spontaneous symmetry breaking, we have

$$
\rho_{i}=\lambda_{i}\langle\omega\rangle,
$$

with $\langle\omega\rangle$ being the related vacuum expectation value.

The above symmetry breaking will result in a massless Goldstone boson which couples to the $\delta$ and $\xi$ scalars as well as the SM leptons. However, these couplings are suppressed by the symmetry breaking scale $\langle\omega\rangle$. For a large symmetry breaking scale $\langle\omega\rangle \gg\langle\phi\rangle \simeq$ $174 \mathrm{GeV}$, the Goldstone will decouple at a very high temperature $T_{\langle\omega\rangle} \gg\langle\phi\rangle$ at which the relativistic degrees of freedom should be about $g_{*}=112.5$ (the SM fields plus the charged scalars $\delta$ and $\xi$ as well as the singlet fermion $N_{R}$ ). The temperature of the Goldstone at the BBN epoch $T \sim 1 \mathrm{MeV}$ thus should be [41]

$$
\left(\frac{T_{\langle\omega\rangle}}{T}\right)^{4} \simeq\left(\frac{10.75}{112.5}\right)^{\frac{4}{3}} \simeq 0.044 .
$$

Therefore, the Goldstone will give a negligible contribution to the effective number of additional light neutrinos [41], i.e.

$$
\Delta N_{\nu}=\frac{4}{7}\left(\frac{T_{\langle\omega\rangle}}{T}\right)^{4} \simeq 0.025 .
$$

\section{$3 \quad$ Neutrino masses and dark matter}

As shown in figure 1, the non-SM scalars and fermions can mediate a three-loop diagram in association with the Yukawa couplings for generating the SM lepton masses. Clearly, after the electroweak symmetry breaking, this three-loop diagram will contribute a Majorana mass term of the left-handed neutrinos. Since the real singlet scalars $\sigma$ are very heavy, they can be integrated out from eq. (2.3). The resulting Lagrangian then can contain a sizable quartic coupling between the singly charged scalars $\delta$ and $\xi$, i.e.

$$
\mathcal{L} \supset-\kappa\left(\delta^{\dagger} \xi\right)^{2}+\text { H.c. with } \kappa=\sum_{i} \frac{\rho_{i}^{2}}{M_{\sigma_{i}}^{2}} .
$$

We hence obtain the KNT model where the lightest one of the Majorana fermions $N_{i}$ can be a stable dark matter particle. For simplicity, we will not repeat the details of the neutrino masses and the dark matter $[32,33,40]$. 


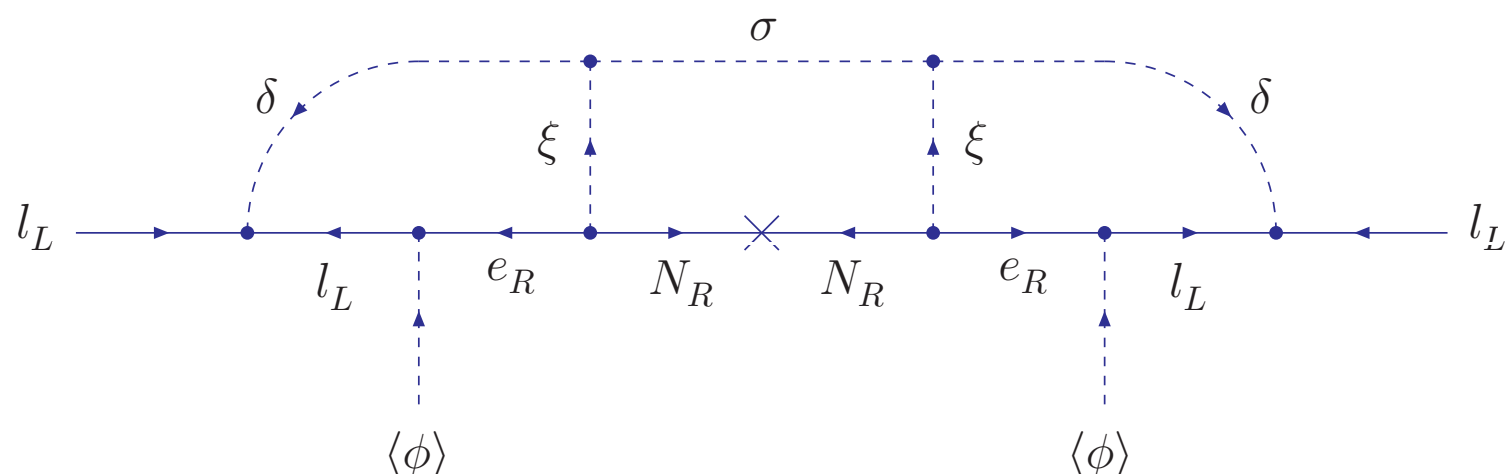

Figure 1. The three-loop diagram for neutrino mass generation.

\section{Leptogenesis}

Figure 2 shows the decays of the real singlet scalars $\sigma_{i}$ as well as the decays of the singly charged scalar pairs $\left(\delta, \delta^{*}\right)$ and $\left(\xi, \xi^{*}\right)$. According to the lepton number assignment (2.1), the real scalar decays can generate a lepton asymmetry stored in the charged scalars. Through the charged scalar decays, the SM leptons then can acquire a lepton asymmetry, which participates in the sphaleron processes so that it can be partially converted to a baryon asymmetry. We calculate the width in the real scalar decays at tree level,

$$
\Gamma_{\sigma_{i}}=\Gamma\left(\sigma_{i} \rightarrow \delta^{*}+\xi\right)+\Gamma\left(\sigma_{i} \rightarrow \delta+\xi^{*}\right)=\frac{1}{8 \pi} \frac{\left|\rho_{i}\right|^{2}}{M_{\sigma_{i}}},
$$

and the CP asymmetry at one-loop order,

$$
\begin{aligned}
\varepsilon_{\sigma_{i}} & =\frac{\Gamma\left(\sigma_{i} \rightarrow \delta^{*}+\xi\right)-\Gamma\left(\sigma_{i} \rightarrow \delta+\xi^{*}\right)}{\Gamma_{\sigma_{i}}} \\
& =\frac{1}{8 \pi} \frac{\operatorname{Im}\left(\rho_{i}^{* 2} \rho_{j}^{2}\right)}{\left|\rho_{i}\right|^{2}} \frac{1}{M_{\sigma_{j}}^{2}-M_{\sigma_{i}}^{2}} \\
& =\frac{\sin 2 \alpha_{j i}}{8 \pi} \frac{\left|\rho_{j}\right|^{2}}{M_{\sigma_{j}}^{2}-M_{\sigma_{i}}^{2}} \text { with } \alpha_{j i}=\arg \left(\frac{\rho_{j}}{\rho_{i}}\right) .
\end{aligned}
$$

As an example, we assume the real scalar $\sigma_{1}$ much lighter than the other one $\sigma_{2}$. The final baryon asymmetry then should mainly come from the $\sigma_{1}$ decays. For a numerical estimation, we define

$$
K=\left.\frac{\Gamma_{\sigma_{1}}}{2 H(T)}\right|_{T=M_{\sigma_{1}}},
$$

where $H(T)$ is the Hubble constant,

$$
H=\left(\frac{8 \pi^{3} g_{*}}{90}\right)^{\frac{1}{2}} \frac{T^{2}}{M_{\mathrm{Pl}}},
$$

with $g_{*}$ being the relativistic degrees of freedom during the leptogenesis epoch. In the strong washout region where

$$
1 \ll K \lesssim 10^{6}
$$



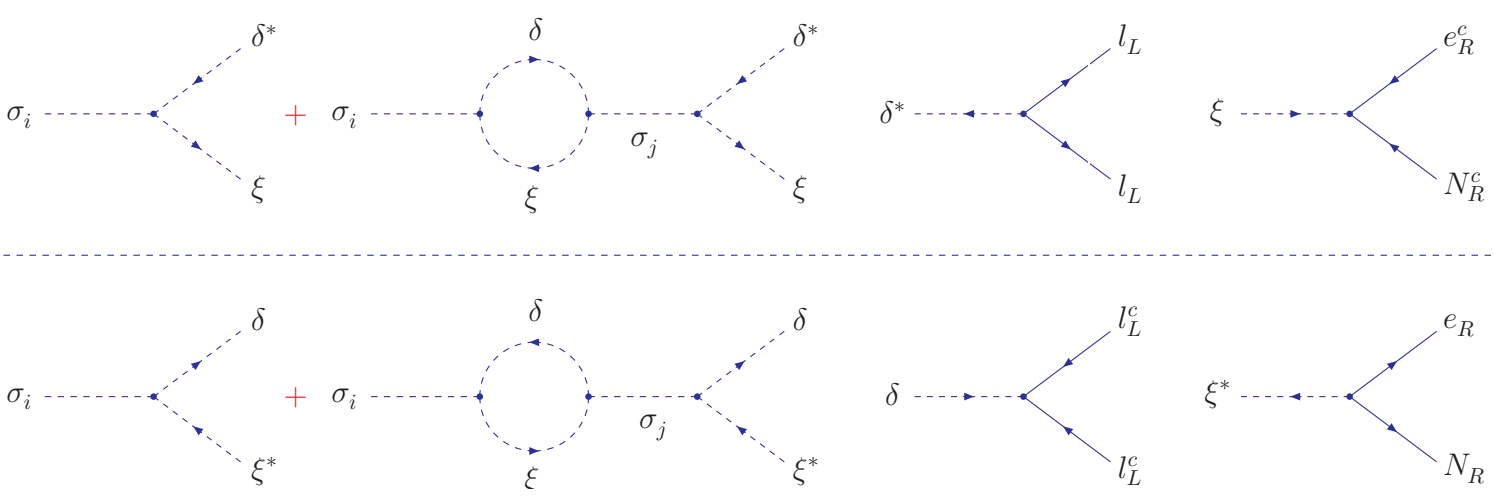

Figure 2. The real scalars $\sigma_{i}$ decay into the charged scalars $\delta$ and $\xi$ which subsequently decay into the SM leptons $l_{L}$ and $e_{R}$ as well as the fermion singlets $N_{R}$.

the final baryon asymmetry can be simply described by [41]

$$
\begin{aligned}
\eta_{B} & =\frac{n_{B}}{s} \simeq-\frac{28}{79} \times \frac{\varepsilon_{\sigma_{1}}}{g_{*} K z_{f}} \\
\text { with } z_{f} & =\frac{M_{\sigma_{1}}}{T_{f}} \simeq 4.2(\ln K)^{0.6} .
\end{aligned}
$$

Here $n_{B}$ and $s$, respectively, are the baryon number density and the entropy density, while the factor $-\frac{28}{79}$ is the sphaleron lepton-to-baryon coefficient [42]. After fixing $g_{*}=112.5$ (the SM fields plus two singly charged scalars as well as two singlet fermions) and inputting,

$$
M_{\sigma_{1}}=\left|\rho_{1}\right|=10^{14} \mathrm{GeV}, \quad M_{\sigma_{2}}=\left|\rho_{2}\right|=10^{15} \mathrm{GeV},
$$

we read

$$
K=137, \quad z_{f}=11, \quad T_{f}=9 \times 10^{12} \mathrm{GeV}, \quad \varepsilon_{\sigma_{1}}=5 \times 10^{-5}\left(\frac{\sin 2 \alpha_{21}}{1.25 \times 10^{-3}}\right) .
$$

The baryon asymmetry then can arrive at an expected value,

$$
\eta_{B}=10^{-10}\left(\frac{\sin 2 \alpha_{21}}{1.25 \times 10^{-3}}\right) .
$$

Ones may worry about the produced lepton asymmetry will be erased by some leptonnumber-violating processes at low energies since figure 1 actually results in the dimension- 5 Weinberg operators violating the lepton number by two units. Usually ones estimate these processes will decouple at a very high temperature [43],

$$
T=10^{12} \mathrm{GeV}\left[\frac{0.04 \mathrm{eV}^{2}}{\operatorname{Tr}\left(m_{\nu}^{\dagger} m_{\nu}\right)}\right],
$$

with $m_{\nu}$ being the Majorana neutrino mass matrix. Therefore, no lepton asymmetry can survive above the temperature $T \sim 10^{12} \mathrm{GeV}$ if the neutrino masses arrives at an acceptable level. In our model, the effective dimension- 5 operators are induced by integrating out the 
scalars $\sigma, \delta$ and $\xi$ as well as the fermions $N_{R}$. However, the fields $\delta, \xi$ and $N_{R}$ are near the $\mathrm{TeV}$ scale, i.e. their masses are lighter than the crucial temperature $T \sim 10^{12} \mathrm{GeV}$. So, the estimation (4.10) is not consistent with the present scenario. Actually, in the above demonstration, the cubic terms among the scalars $\sigma, \delta$ and $\xi$ are assumed to provide the unique source of the lepton number violation. After this lepton number violation is decoupled, no other lepton-number-violating processes can keep in equilibrium to wash out the produced lepton asymmetry.

If we take the lepton number assignment in the KNT model, the Majorana mass term of the singlet fermions $N_{R}$ should be the unique source of the lepton number violation. In this case, the lepton asymmetry in the singly charged scalar $\xi$ will get converted to a lepton asymmetry in the SM leptons $e_{R}$ and an equal lepton asymmetry in the singlet fermions $N_{R}$. The $N_{R}-N_{R}^{c}$ oscillations will wash out the $N_{R}$ asymmetry but will not affect the $e_{R}$ asymmetry. So, the lepton asymmetry in the SM leptons $e_{R}$ can survive for a successful leptogenesis. This SM lepton asymmetry definitely equals to that in the case with the lepton number assignment (2.1).

Ones can analyze the chemical potentials [42] to understand the leptogenesis in the present model. For this purpose, we denote $\mu_{q, d, u}$ for the chemical potentials of the SM quarks $q_{L}\left(3,2,+\frac{1}{6}\right), d_{R}\left(3,1,-\frac{1}{3}\right)$ and $u_{R}\left(3,1,+\frac{2}{3}\right)$, while $\mu_{l, e, \phi, N, \delta, \xi}$ for the chemical potentials of the SM leptons and Higgs scalar $l_{L}, e_{R}, \phi$ as well as the non-SM fields $N_{R}, \delta, \xi$. The singly charged scalars $\delta$ and $\xi$ as well as the singlet fermions $N_{R}$ are assumed near the $\mathrm{TeV}$ scale. Therefore, above the TeV scale, the SM Yukawa interactions yield [42],

$$
\begin{aligned}
-\mu_{q}+\mu_{\phi}+\mu_{d} & =0, \\
-\mu_{q}-\mu_{\phi}+\mu_{u} & =0, \\
-\mu_{l}+\mu_{\phi}+\mu_{e} & =0,
\end{aligned}
$$

the sphalerons constrain [42],

$$
3 \mu_{q}+\mu_{l}=0,
$$

while the vanishing hypercharge in the universe require,

$$
3\left[\left(\frac{1}{6} \mu_{q} \times 2-\frac{1}{3} \mu_{d}+\frac{2}{3} \mu_{u}\right) \times 3+\left(-\frac{1}{2} \mu_{l} \times 2-\mu_{e}\right)\right]+\left(\frac{1}{2} \mu_{\phi} \times 2+\mu_{\delta}+\mu_{\xi}\right) \times 2=0 .
$$

Furthermore, from the non-SM Yukawa interactions and Majorana masses in eq. (2.3), we obtain,

$$
\begin{aligned}
\mu_{\delta}+2 \mu_{l} & =0, \\
\mu_{\xi}+\mu_{e}+\mu_{N} & =0, \\
-2 \mu_{N} & =0 .
\end{aligned}
$$

Note in eqs. (4.11)-(4.14), we have identified the chemical potentials of the differentgeneration fermions because the Yukawa interactions establish an equilibrium between the different generations. By solving eqs. (4.11)-(4.14), we find

$$
\begin{array}{llll}
\mu_{q}=-\frac{8}{3} \mu_{e}, & \mu_{d}=-\frac{29}{3} \mu_{e}, & \mu_{u}=\frac{13}{3} \mu_{e}, & \mu_{l}=8 \mu_{e}, \\
\mu_{\phi}=7 \mu_{e}, & \mu_{\delta}=-16 \mu_{e}, & \mu_{\xi}=-\mu_{e}, & \mu_{N}=0 .
\end{array}
$$


Clearly, the singlet fermions have a zero chemical potential because of their Majorana masses. However, the Majorana masses of the singlet fermions will not wash out the baryon/lepton asymmetry in the SM quarks/leptons as well as the lepton asymmetry in the singly charged scalars $\delta$ and $\xi$. Below the TeV scale, the non-relativistic $\delta$ and $\xi$ have decayed so that the condition (4.13) for the zero hypercharge should be modified by [42],

$$
3\left(\mu_{q}-\mu_{d}+2 \mu_{u}-\mu_{l}-\mu_{e}\right)+2 \mu_{\phi}=0 .
$$

Ones then can solve eqs. (4.11), (4.12) and (4.16) to determine the chemical potentials as below [42],

$$
\mu_{q}=-\frac{7}{9} \mu_{e}, \quad \mu_{d}=-\frac{19}{9} \mu_{e}, \quad \mu_{u}=\frac{5}{9} \mu_{e}, \quad \mu_{l}=\frac{7}{3} \mu_{e}, \quad \mu_{\phi}=\frac{4}{3} \mu_{e} .
$$

The relation between the baryon and lepton numbers then should be [42]

$$
B=\frac{28}{79}(B-L) \quad \text { with } B=-\frac{28}{3} \mu_{e}, \quad L=\frac{51}{3} \mu_{e} .
$$

\section{Summary}

In this paper we have simultaneously realized a high-scale leptogenesis and a low-scale neutrino mass generation. Specifically we have introduced two real singlet scalars to the KNT model which extended the SM by two singly charged scalars and two or more singlet fermions. The real scalar decays and then the charged scalar decays can produce a lepton asymmetry stored in the SM leptons. This lepton asymmetry can be partially converted to a baryon asymmetry by the sphaleron processes. At the low energy scales, we can integrate out the real scalars to derive the KNT model, where the neutrino masses are induced at three-loop level while the dark matter particle is given by the lightest singlet fermion. For the variant KNT models [36, 37], we can consider two real triplet or quintuplet scalars.

\section{Acknowledgments}

The author was supported by the National Natural Science Foundation of China under Grant No. 11675100, the Recruitment Program for Young Professionals under Grant No. 15Z127060004, the Shanghai Jiao Tong University under Grant No. WF220407201 and the Shanghai Laboratory for Particle Physics and Cosmology under Grant No. 11DZ2260700. This work was also supported by the Key Laboratory for Particle Physics, Astrophysics and Cosmology, Ministry of Education.

Open Access. This article is distributed under the terms of the Creative Commons Attribution License (CC-BY 4.0), which permits any use, distribution and reproduction in any medium, provided the original author(s) and source are credited. 


\section{References}

[1] Particle Data Group collaboration, C. Patrignani et al., Particle Data Group, Chin. Phys. C 40 (2016) 1000001.

[2] P. Minkowski, $\mu \rightarrow e \gamma$ at a Rate of One Out of $10^{9}$ Muon Decays?, Phys. Lett. B 67 (1977) 421 [INSPIRE].

[3] T. Yanagida, Horizontal Symmetry And Masses Of Neutrinos, in Proceedings of the Workshop on Unified Theory and the Baryon Number of the Universe, O. Sawada and A. Sugamoto eds., KEK, Tsukuba, Japan 1979, p. 95.

[4] M. Gell-Mann, P. Ramond and R. Slansky, Complex Spinors and Unified Theories, in Supergravity, F. van Nieuwenhuizen and D. Freedman eds., North Holland, Amsterdam, The Netherlands (1979), p. 315.

[5] S.L. Glashow, The Future of Elementary Particle Physics, in Quarks and Leptons, M. Lévy et al. eds., Plenum, New York, U.S.A. (1980), p. 707.

[6] R.N. Mohapatra and G. Senjanović, Neutrino Mass and Spontaneous Parity Violation, Phys. Rev. Lett. 44 (1980) 912 [INSPIRE].

[7] M. Magg and C. Wetterich, Neutrino Mass Problem and Gauge Hierarchy, Phys. Lett. B 94 (1980) 61 [INSPIRE].

[8] J. Schechter and J.W.F. Valle, Neutrino Masses in $\mathrm{SU}(2) \times \mathrm{U}(1)$ Theories, Phys. Rev. D 22 (1980) 2227 [INSPIRE].

[9] T.P. Cheng and L.-F. Li, Neutrino Masses, Mixings and Oscillations in $\mathrm{SU}(2) \times \mathrm{U}(1)$ Models of Electroweak Interactions, Phys. Rev. D 22 (1980) 2860 [InSPIRE].

[10] G. Lazarides, Q. Shafi and C. Wetterich, Proton Lifetime and Fermion Masses in an $\mathrm{SO}(10)$ Model, Nucl. Phys. B 181 (1981) 287 [InSPIRE].

[11] R.N. Mohapatra and G. Senjanović, Neutrino Masses and Mixings in Gauge Models with Spontaneous Parity Violation, Phys. Rev. D 23 (1981) 165 [InSPIRE].

[12] R. Foot, H. Lew, X.G. He and G.C. Joshi, Seesaw Neutrino Masses Induced by a Triplet of Leptons, Z. Phys. C 44 (1989) 441 [InSPIRE].

[13] E. Ma, Pathways to naturally small neutrino masses, Phys. Rev. Lett. 81 (1998) 1171 [hep-ph/9805219] [INSPIRE].

[14] M. Fukugita and T. Yanagida, Baryogenesis Without Grand Unification, Phys. Lett. B 174 (1986) 45 [INSPIRE].

[15] P. Langacker, R.D. Peccei and T. Yanagida, Invisible Axions and Light Neutrinos: Are They Connected?, Mod. Phys. Lett. A 1 (1986) 541 [InSPIRE].

[16] M.A. Luty, Baryogenesis via leptogenesis, Phys. Rev. D 45 (1992) 455 [InSPIRE].

[17] R.N. Mohapatra and X. Zhang, Electroweak baryogenesis in left-right symmetric models, Phys. Rev. D 46 (1992) 5331 [INSPIRE].

[18] M. Flanz, E.A. Paschos and U. Sarkar, Baryogenesis from a lepton asymmetric universe, Phys. Lett. B 345 (1995) 248 [Erratum ibid. B 384 (1996) 487] [hep-ph/9411366] [INSPIRE].

[19] M. Flanz, E.A. Paschos, U. Sarkar and J. Weiss, Baryogenesis through mixing of heavy Majorana neutrinos, Phys. Lett. B 389 (1996) 693 [hep-ph/9607310] [INSPIRE]. 
[20] L. Covi, E. Roulet and F. Vissani, CP violating decays in leptogenesis scenarios, Phys. Lett. B 384 (1996) 169 [hep-ph/9605319] [INSPIRE].

[21] A. Pilaftsis, CP violation and baryogenesis due to heavy Majorana neutrinos, Phys. Rev. D 56 (1997) 5431 [hep-ph/9707235] [INSPIRE].

[22] E. Ma and U. Sarkar, Neutrino masses and leptogenesis with heavy Higgs triplets, Phys. Rev. Lett. 80 (1998) 5716 [hep-ph/9802445] [INSPIRE].

[23] R. Barbieri, P. Creminelli, A. Strumia and N. Tetradis, Baryogenesis through leptogenesis, Nucl. Phys. B 575 (2000) 61 [hep-ph/9911315] [INSPIRE].

[24] S. Davidson and A. Ibarra, A Lower bound on the right-handed neutrino mass from leptogenesis, Phys. Lett. B 535 (2002) 25 [hep-ph/0202239] [INSPIRE].

[25] G.F. Giudice, A. Notari, M. Raidal, A. Riotto and A. Strumia, Towards a complete theory of thermal leptogenesis in the SM and MSSM, Nucl. Phys. B 685 (2004) 89 [hep-ph/0310123] [INSPIRE].

[26] W. Buchmüller, P. Di Bari and M. Plümacher, Leptogenesis for pedestrians, Annals Phys. 315 (2005) 305 [hep-ph/0401240] [INSPIRE].

[27] S. Davidson, E. Nardi and Y. Nir, Leptogenesis, Phys. Rept. 466 (2008) 105 [arXiv:0802.2962] [INSPIRE].

[28] A. Zee, A Theory of Lepton Number Violation, Neutrino Majorana Mass and Oscillation, Phys. Lett. B 93 (1980) 389 [Erratum ibid. B 95 (1980) 461] [INSPIRE].

[29] A. Zee, Charged Scalar Field and Quantum Number Violations, Phys. Lett. B 161 (1985) 141 [INSPIRE].

[30] K.S. Babu, Model of 'Calculable' Majorana Neutrino Masses, Phys. Lett. B 203 (1988) 132 [INSPIRE].

[31] K.S. Babu and C.N. Leung, Classification of effective neutrino mass operators, Nucl. Phys. B 619 (2001) 667 [hep-ph/0106054] [INSPIRE].

[32] L.M. Krauss, S. Nasri and M. Trodden, A model for neutrino masses and dark matter, Phys. Rev. D 67 (2003) 085002 [hep-ph/0210389] [INSPIRE].

[33] K. Cheung and O. Seto, Phenomenology of TeV right-handed neutrino and the dark matter model, Phys. Rev. D 69 (2004) 113009 [hep-ph/0403003] [INSPIRE].

[34] E. Ma, Verifiable radiative seesaw mechanism of neutrino mass and dark matter, Phys. Rev. D 73 (2006) 077301 [hep-ph/0601225] [INSPIRE].

[35] S. Kanemura, O. Seto and T. Shimomura, Masses of dark matter and neutrino from TeV scale spontaneous $\mathrm{U}(1)_{B-L}$ breaking, Phys. Rev. D 84 (2011) 016004 [arXiv:1101.5713] [INSPIRE].

[36] A. Ahriche, C.-S. Chen, K.L. McDonald and S. Nasri, Three-loop model of neutrino mass with dark matter, Phys. Rev. D 90 (2014) 015024 [arXiv: 1404.2696] [INSPIRE].

[37] A. Ahriche, K.L. McDonald and S. Nasri, A Model of Radiative Neutrino Mass: with or without Dark Matter, JHEP 10 (2014) 167 [arXiv:1404.5917] [INSPIRE].

[38] E. Ma, Derivation of Dark Matter Parity from Lepton Parity, Phys. Rev. Lett. 115 (2015) 011801 [arXiv: 1502.02200] [INSPIRE]. 
[39] P.-H. Gu, E. Ma and U. Sarkar, Connecting Radiative Neutrino Mass, Neutron-Antineutron Oscillation, Proton Decay and Leptogenesis through Dark Matter, Phys. Rev. D 94 (2016) 111701 [arXiv: 1608.02118] [INSPIRE].

[40] L. Bergstrom, The 130 GeV Fingerprint of Right-Handed Neutrino Dark Matter, Phys. Rev. D 86 (2012) 103514 [arXiv: 1208.6082] [INSPIRE].

[41] E.W. Kolb and M.S. Turner, The Early Universe, Addison-Wesley, (1990).

[42] J.A. Harvey and M.S. Turner, Cosmological baryon and lepton number in the presence of electroweak fermion number violation, Phys. Rev. D 42 (1990) 3344 [INSPIRE].

[43] M. Fukugita and T. Yanagida, Sphaleron Induced Baryon Number Nonconservation and a Constraint on Majorana Neutrino Masses, Phys. Rev. D 42 (1990) 1285 [INSPIRE]. 